\title{
A case report of acute respiratory failure after ultrasound- Guided supraclavicular block
}

\author{
Lim MingJian ${ }^{1}$, Nithiyananthan Muruganath ${ }^{1}$, Abirami Ramanathan ${ }^{2}$ and Suhitharan Thangavelautham ${ }^{1 *}$ \\ ${ }^{1}$ Department of Anaesthesia, Singapore General Hospital, Singapore \\ ${ }^{2}$ Ngee Ann Ploytechnic, Singapore
}

\begin{abstract}
This paper presents a case of acute respiratory failure after a successful Ultrasound guided supraclavicular brachial plexus block. Through this report we wish to highlight the importance of a thorough pre-operative assessment to identify high risk patients and close monitoring after the block for such complications to reduce adverse outcomes. Reducing the local anesthetic volume, ultrasound guided techniques and infra-clavicular or axillary brachial plexus blocks should be considered in high risk patients.
\end{abstract}

\section{Introduction}

The supraclavicular brachial plexus block is a commonly used regional anesthetic technique for patients undergoing upper limb surgeries where the block is performed at C5-T1 nerve roots of the brachial plexus trunks. Ultrasound guided techniques allow more precise delivery of local anesthetic injection with lower volumes to achieve the desired surgical block and has been associated with lower incidence of complications [1]. Despite the safety of ultrasound guided blocks respiratory complications could still happen in patients with multiple risk factors [2]. We report a case with unrecognized risk factors who developed acute respiratory distress requiring tracheal intubation after a supraclavicular bock.

\section{Case report}

A 69-year-old female, $147 \mathrm{~cm} 46 \mathrm{~kg}$ BMI 21, with hypertensive nephropathy leading to end stage renal failure requiring dialysis presented for a right brachio-basilic arteriovenous fistula creation. On pre-operative assessment she denied any history of asthma or chronic obstructive pulmonary disease. She had mild cough with whitish sputum 3 days ago without any fever, myalgia, chest pain or sore throat. On examination she was well, no chest signs identified and oxygen saturation on room air was $98 \%$. Routine monitoring established and a right supraclavicular block was performed uneventfully in supine position using Stimuplex $50 \mathrm{~mm} 22 \mathrm{G}$ needle. $0.75 \%$ Ropivacaine $12 \mathrm{ml}$ and $2 \%$ Lignocaine $6 \mathrm{ml}$ was injected around the cords identified under ultrasound guidance and the good spread of local anesthetic around the codes identified.

Within 5 minutes of completion of block, the patient experienced sudden onset of shortness of breath hoarseness of voice and anxiety. On examination, she had poor respiratory efforts, bilateral rhonchi with poor air entry and her $\mathrm{SpO}_{2}$ fell to $80 \%$ on room air. She was propped up and administered Salbutamol, Ipratropium nebulization followed by IV ketamine $10 \mathrm{mg}$ which gave a transient improvement of her symptoms. However, she still had significantly impaired respiratory efforts with hypoxia and hypercarbia. Trachea was intubated with rapid sequence induction and positive pressure ventilation was initiated.
Post intubation chest X-ray showed an underlying right lower zone consolidation with effusion without any pneumothorax or elevation of the right hemi-diaphragm. Surgery was abandoned due the unexpected complication and the patient was moved to post-anesthetic care unit (PACU). After the reversal of muscle blockade, she had low tidal volumes on spontaneous ventilation and was assisted with on pressure support ventilation.

Four hours later, the block has worn off and she regained some sensation and motor power in her right arm. Her respiratory efforts have also improved at the same time and she was successfully weaned off pressure support ventilation and extubated.

\section{Discussion}

Acute respiratory distress after supraclavicular brachial plexus block has been reported due to phrenic nerve palsy (PNP), pneumothorax, recurrent laryngeal nerve palsy or development of bronchospasm.

Hemidiaphragmatic paralysis due to inadvertent PNP is a wellrecognized complication of brachial plexus blocks and has been reported to be as high as and 50\% to 67\% [3]. Phrenic nerve arises from roots of C3, C4 and C5, and its involvement occurs in supraclavicular block because of its anatomical relation with brachial plexus and direct infiltration of local anaesthetic on the nerve or cephalic spread of local anaesthetic. Furthermore a cadaveric study showed that an accessory phrenic nerve may arise from C5 and C6 roots or from the nerve to the subclavius muscle up to $75 \%$ [4]. Blocking the accessory phrenic nerve may lead to partial block of the hemidiaphragm and is consistent with the outcomes of the study by Neal and colleagues, especially the preservation of Forced vital capacity with supraclavicular block even in those with evidence of hemiparesis of the diaphragm [5]. Moreover despite the higher incidence of PNP and hemidiaphragmatic paralysis,

*Correspondence to: Suhitharan Thangavelautham, Department of Anaesthesia, Singapore General Hospital, Singapore, E-mail: tsuhitharan@yahoo.com

Received: July 28, 2018; Accepted: August 16, 2018; Published: August 20, 2018 
healthy patients remain asymptomatic at rest since the other respiratory muscles compensate the ipsilateral hemidiaphragmatic palsy [6].

Acute bronchospasm after supraclavicular block is rare. To our knowledge there is just one case report by Bhat Pai et al that documented this complication [7]. It is believed that the spread of local anaesthetic caused sympathetic blockade of the T1-4 segments of the sympathetic chain causing an imbalance between the sympathetic and parasympathetic nervous systems supplying the bronchi. Other reports of acute bronchospasm after brachial plexus block were only with an interscalene approach. Recurrent laryngeal nerve palsy presents with hoarseness of voice and respiratory distress and reported to occur in $1.3 \%$ of the patients after right sided subclavian perivascular block [8]. This complication has only been reported on the right side and proposed mechanism is that the local anesthetic travels along the subclavian artery to the place where the nerve loops around it [9].

In our patient, we believe that the cause of respiratory distress was multifactorial in nature. Firstly her ability to compensate hemidiaphragamatic palsy caused by PNP was significantly reduced due to the underlying pneumonia and pleural effusions which were affecting the gas exchange. Secondly bilateral bronchospasm would have reduced the oxygen delivery and increased the oxygen demand due to increased work of breathing and attributed further her hypoxia. Finally right recurrent laryngeal nerve palsy would have caused additional stress and further aggravating her acute respiratory distress. Moreover chronic renal failure itself may be a risk factor. A case series consists of three patients undergoing arteriovenous graft placement with supraclavicular block developed respiratory arrest necessitating tracheal intubation in patients; however, the exact underlying causes other than chronic renal failure and obesity could not be delineated. The authors suggested that chronic renal failure may represent a highrisk group for respiratory failure after supraclavicular brachial plexus block [10].

Chest $\mathrm{X}$ ray done after intubation (Figure 1) excluded the pneumothorax, however it did not demonstrate elevated right hemi

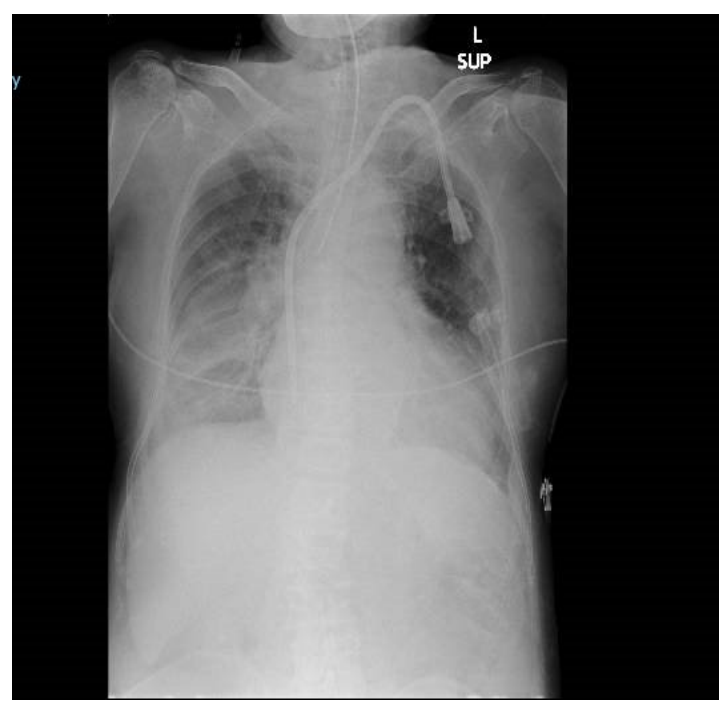

Figure 1. Chest $\mathrm{X}$ ray done after intubation diaphragm since it was taken immediately after intubation which required muscle relaxation with Rocuronium. Bronchospasm was confirmed clinically by auscultation and improvement of wheeze after bronchodilators. Recurrent laryngeal nerve involvement was possible due to hoarse voice and dyspnea but we could not demonstrate any vocal cord asymmetry during intubation due to the effect of muscle relaxant.

From our experience in this case, we have learnt that respiratory distress following supraclavicular block is multifactorial in nature and it could happen even in low volume block done under ultrasound guidance in patients with limited respiratory reserves and risk factors. Hence a through pre-operative assessment to identify high risk patients and close monitoring after the block for such complications are vital to reduce the adverse outcomes. Alternative approaches with minimal risk of phrenic nerve, recurrent laryngeal nerve palsies or development of pneumothorax, such as infraclavicular or axillary brachial plexus block should be considered in patients with known risk factors.

\section{Conclusion}

Symptomatic diaphragmatic paralysis following supraclavicular block is common especially in patients with limited respiratory reserves. Hence a thorough pre-operative assessment to identify high risk patients and close monitoring after the block for such complications are vital to reduce the adverse outcomes. Reducing the local anesthetic volume, ultrasound guided techniques and infra-clavicular or axillary brachial plexus blocks should be considered in high risk patients.

\section{Acknowledgement}

Published with consent of the patient.

\section{References}

1. Renes SH, Spoormans HH, Gielen MJ, Rettig HC, van Geffen GJ (2009) Hemidiaphragmatic paresis can be avoided in ultrasound-guided supraclavicular brachial plexus block. Reg Anesth Pain Med 34: 595-599. [Crossref]

2. Guirguis M, Karroum R, Abd-Elsayed AA, Mounir-Soliman L (2012) Acute respiratory distress following ultrasound-guided supraclavicular block. Ochsner J 12: 159-162. [Crossref]

3. Pham-Dang C, Gunst JP, Gouin F, Poirier P, Touchais S, et al. (1997) A nove supraclavicular approach to brachial plexus block. Anesth Analg 85: 111-116. [Crossref]

4. Bergman RA, Thompson SA, Afifi AK, Saadeh FA (1988) Compendium of Human Anatomic Variation. Baltimore: Urban \& Schwarzenberg: 138-139.

5. Neal JM, Moore JM, Kopacz DJ, Liu SS, Kramer DJ, et al. (1998) Quantitative analysis of respiratory, motor, and sensory function after supraclavicular block. Anesth Analg 86: 1239-1244. [Crossref]

6. Mak PH, Irwin MG, Ooi CG, Chow BF (2001) Incidence of diaphragmatic paralysis following supraclavicular block and its effects on pulmonary function. Anaesthesia 56: 350-369. [Crossref]

7. Rohini V Bhat Pai, Harihar Hegde V, Santosh MCB, Roopa S (2011) Bronchospasm following supraclavicular brachial plexus block. Anesth Essays Res 5: 211-213. [Crossref]

8. HickeyR, Garland TA, Ramamurthy S (1989) Subclavian perivascular block: influence of location of paresthesia. Anesth Analg 68: 767-771. [Crossref]

9. Winnie AP (1990) Perivascular techniques of brachial plexus block. ( $2^{\text {nd }}$ edn) Philadelphia: WB Saunders. Plexus anesthesia 1: 236-237.

10. Afonso A, Beilin Y (2013) Respiratory arrest in patients undergoing arteriovenous graft placement with supraclavicular brachial plexus block: a case series. $J$ Clin Anesth 25: 321-323. [Crossref]

Copyright: (C2018 Lim MJ. This is an open-access article distributed under the terms of the Creative Commons Attribution License, which permits unrestricted use, distribution, and reproduction in any medium, provided the original author and source are credited. 